\title{
Evaluation of an adaptive learning technology in a first-year extended curriculum programme physics course
}

\author{
Moses Basitere ${ }^{a}$, Eunice Ivala \\ ${ }^{\text {a }}$ Department of Chemical Engineering, Cape Peninsula University of Technology, South Africa \\ ${ }^{b}$ Centre for Innovative Educational Technology, Cape Peninsula University of Technology, South Africa
}

\begin{abstract}
Personalised, adaptive online learning platforms that form part of web-based proficiency tests play a major role in the improvement of the quality of learning in physics and assist learners in building proficiency, preparing for tests and using their time more effectively. In this study, the effectiveness of an adaptive learning platform, Wiley Plus ORION, was evaluated using proficiency test scores compared to paper-based test scores in a first-year introductory engineering physics course. Learners' performance activities on the adaptive learning platform as well as their performance on the proficiency tests and their impact on the paper-based midterm averaged test were investigated using both qualitative and quantitative methods of data collection. A comparison between learners' performance on the proficiency tests and a paper-based midterm test was done to evaluate whether there was a correlation between their performance on the proficiency tests and the midterm test. Focus group interviews were carried out with three categories of learners to elicit their experiences. Results showed that there was a positive relationship between high-performing learners' proficiency score in the midterm averaged test and that the proficiency test enhanced learners' performance in the paper-based midterm averaged test.
\end{abstract}

Keywords: adaptive, metacognitive report, online assessment, productivity reports, Wiley Plus ORION

Categories: • Human-centred computing $\sim$ Interaction design theory, concepts and paradigms $\bullet$ Applied computing $\sim$ Education $\bullet$ Applied computing $\sim$ Computer-managed instruction $\bullet$ Applied computing $\sim$ Collaborative learning

\section{Email:}

Moses Basitere basiterem@cput.ac.za (CORRESPONDING),

Eunice Ivala ivalae@cput.ac.za
Article history:

Received: 01 Mar 2017

Accepted: 21 Sep 2017

Available online: 08 Dec 2017

\section{INTRODUCTION}

The success of learner performance in courses such as mathematics and physics largely relies on educators monitoring and providing timeous individualised feedback to their learners. Timeous feedback to learners can enhance learning as they act on the feedback provided (Hattie \& Timperley, 2007; Shute, 2008). Nicol (2011) argues that a lecturer's meaningful feedback needs to be easy for learners

Basitere, M. and Ivala, E. (2017). Evaluation of an adaptive learning technology in a first-year extended curriculum programme physics course. South African Computer Journal 29(3), 1-15. https://doi.org/10.18489/sacj.v29i3.476 Copyright (C) the author(s); published under a Creative Commons NonCommercial 4.0 License (CC BY-NC 4.0). SACJ is a publication of the South African Institute of Computer Scientists and Information Technologists. ISSN 1015-7999 (print) ISSN 2313-7835 (online). 
to understand, timely, non-judgmental, contextualised, broad minded, balanced, forward looking and transferable. Despite the value of a lecturer's feedback to learners, providing individualised feedback to learners is nearly impossible to achieve due to a lack of time, as lecturers spend most of their time in the classroom lecturing, due to the high number of learners in the classroom, a lack of qualified tutors or lecturer assistants, and the fact that individualised feedback is time consuming (Basitere \& Ivala, 2015; Gibbs, 2010). As a result of these challenges, educators are seeking innovative ways of providing immediate individualised feedback to learners using online-based learner feedback systems.

One such online-based learner feedback system is the Wiley Plus ORION (WPO) adaptive learning platform, which provides an online platform for learners to access and submit web-based formative assessments, grade them and provide individualised feedback (Basitere \& Ivala, 2015). The adoption of such computerised online systems that provide learners with formative assignments and instantly grade and provide individualised feedback is being seen as one way to effectively improve lecturers' timeous feedback to learners. Furthermore, the online system also provides other benefits, such as grade accessibility using an electronic platform, quantifying and promoting student learning, and also monitoring at-risk learners on the course (Jones, 2008, 1).

John Wiley and Son introduced the WPO adaptive online learning platform in 2014 and it is adapted to meet each individual learner's needs. Learners are allowed to complete diagnostic questions to evaluate their initial level of proficiency in the section being taught in the classroom. The diagnostic test is in the form of randomised multiple-choice questions and the difficulty level of questions is determined by learners' achievement in the diagnostic test. The difficulty level of the diagnostic practice test questions presented to learners is adapted by the online system to match their level of performance. Learners are provided with reports as a guide to their progress during their study and to show their level of performance. Study time data is provided to the learner, broken into specific subject aspects and displayed in a red (low performance), yellow (medium performance) or green (high performance) level and percentage proficiency. The teacher has access to this information and can use data from these reports to design and provide the necessary support to learners.

This paper presents findings of a study carried out in 2015 amongst first-year chemical engineering learners studying Physics at a South African university of technology, and is aimed at evaluating the effectiveness of the WPO adaptive learning platform system in improving learners' learning. The use of this adaptive learning platform was compulsory for all learners who enrolled for the Physics 1 course in the Chemical Engineering Extended Curriculum Programme (ECP). The diagnostic tests were provided to learners to engage with as part of the curriculum and weighed $10 \%$ of the semester score. The study was done in one semester and learners were awarded marks as a way of encouraging them to use the system.

The intervention on this study was based on Laurilland's educational media conversational framework in order to help the researchers understand the effectiveness of the adaptive learning platform through the use of learners' proficiency tests in supporting the effective learning types inspired by feedback-reflection-adaptation phases, in a chemical engineering Physics 1 course. Adaptive learning was evaluated through experiential tasks such as web-based proficiency homework 
(WBPH) and assignments. In this study, three reports generated by the WPO platform were used to evaluate the performance of the online platform in supporting and enhancing student learning. The first report was the metacognitive feedback report, which compares learners' proficiency on the adaptive learning platform to their confidence level. The second report was the productivity feedback report, which compares time spent in the adaptive learning platform by learners to their overall proficiency in the adaptive learning platform assessments. The third report was the most challenging activities feedback, which sorts objectives and chapters by proficiency, from lowest to highest. A comparison between learners' performance on the proficiency tests and the paper-based midterm test was done to evaluate if there was a correlation between learners' performance on the proficiency tests and the paper-based midterm averaged test.

\section{LITERATURE}

Personalised adaptive learning platforms like WPO help learners to improve their level of proficiency on those chapters that are being taught and also assist learners in managing their study time effectively (Schreiber, Smith, \& Getz, 2014). The use of adaptive learning in the classroom setting has been a common practice used by educators all over the world. The instructor often starts by introducing the theoretical concept to the learners and thereafter assesses how well the learners are following the lesson (Basitere \& Ivala, 2017). Assessing how learners are following the instructor's lesson can be achieved by asking leaners a few questions to initiate discussion and dialogue. Based on the discussion with the learners or body language and facial expressions of the learners, the teacher can clarify any misconceptions or elaborate on the concept being taught (McLaren, 2013). The instructor can then create an assignment or homework aimed at addressing the area of learners' weaknesses. However, due to the large size of learner classes, and learners with a different starting point in a knowledge area and who learn at different paces, the challenge is to create an optimal learning experience that caters for all learner types (Schreiber et al., 2014). Hence, there is a need for a tool that can assist the instructor in assessing, offering individualised feedback to learners, and promoting self-paced instructions based on learners' needs.

\subsection{Adaptive learning technologies}

Adaptive technology is reported in literature to have been used to support formative assessment using web-based homework to provide self-directed learning and individualised immediate feedback to learners (Beal, Qu, \& Lee, 2008; Koedinger, Anderson, Hadley, \& Mark, 1997, 8). Immediate feedback is beneficial to the instructor for identifying and remediating individual learner deficiencies and misconceptions in the subject content covered and for modifying learning activities (Eliot \& Woolf, 1994) to improve learning. Furthermore, immediate feedback builds learner confidence, resulting in an improvement in the metacognitive awareness of how learners learn (S. Bonham, Beichner, \& Deardorff, 2001; S. W. Bonham, Deardorff, \& Beichner, 2003).

Furthermore, literature shows that the use of adaptive learning technologies has been reported to lead to an improvement in learners' learning across a wide range of disciplines, such as mathematics 
(Beal et al., 2008; Koedinger et al., 1997, 8), science (Vanlehn et al., 2005), medicine (Eliot \& Woolf, 1994) and computer programming (Corbett \& Anderson, 1992). Several studies have reported on the impact of adopting adaptive media in courses such as physics (S. W. Bonham et al., 2003) and introductory accounting (Jones, 2008, 1). Both S. W. Bonham et al. (2003) and Jones $(2008,1)$ report that adaptive technology in the form of web-based homework as a formative assessment enhanced learning and was as effective as paper-based assessment. Hauk and Segalla (2005) and Demirci (2007) confirm the same results in a study conducted in 19 college algebra classes using the web-based homework system. The main advantages of using adaptive technology as compared to a paper-based system was the immediate feedback and the fact that the system allowed multiple attempts at solving the problem, which encourages learners to devote more time interacting with the course concepts.

Due to a lack of experienced learner assistants or tutors, universities around the world, including South Africa, are abandoning time-intensive approaches of collecting and grading paper-based homework (Basitere \& Ivala, 2015) and are adopting adaptive technology such as WPO and Web Assign. Hence, the focus of this study.

\subsection{Theoretical framework}

This paper is informed by Laurilland's conversational framework to evaluate the effectiveness of an adaptive learning technology platform (Laudrillard, n.d.; Laurillard, 2013) such as WPO in enhancing teaching and learning. Laurilland's framework is specifically designed to gain an understanding of educational environments. The framework has four important learning components:

1. lecturers' learning outcome/concepts

2. learning environments constructed by the lecturer

3. learners' concepts

4. specific action by the learner

According to Laurillard (2013), higher education is more about acquiring "ways of seeing the world" and higher education is differentiated from other forms of learning by the high cognitive communication strategy required of learners and associated mental activities such as discussion, adaptation, interaction, and reflection (Figure 1).

\subsubsection{Conversation dialogue between the lecturer and the learner}

Conceptualisation of learning outcomes should be mutually accessible and agreed upon by both participants (lecturers and learners). Before teaching the topic, the lecturer should assess learners' prior knowledge of the topic. This is then followed by the lecturer introducing the course knowledge concept, learning outcomes, ideas, and the course theoretical background. Hereafter learners should engage with the course content through dialogue, asking questions and receiving responses from the lecturer and peers. Through this dialogue between the lecturer and the learners, the lecturer clarifies 
or elaborates on the course material. In this study, students were encouraged to read the online textbook on WPO before coming to the classroom and the lecturer asked students questions before teaching so as to assess their prior knowledge. Hereafter the lecturer introduced the theoretical concepts of the chapter being taught and students were allowed to ask questions to gain clarity on what was being taught.

\subsubsection{Lecturer defining the learning environment}

The lecturer must create a conducive learning environment that may include the learning task to be given to the learners in the form of an experiential task such as a WPO proficiency test and web-based homework. The learner is expected to put the theory into practice by performing the experiential tasks provided by the teacher. The teacher continuously monitors the learners' experiential progression and provides appropriate feedback, which enables learners to improve their understanding of the course material (Laudrillard, n.d.; Laurillard, 2013).

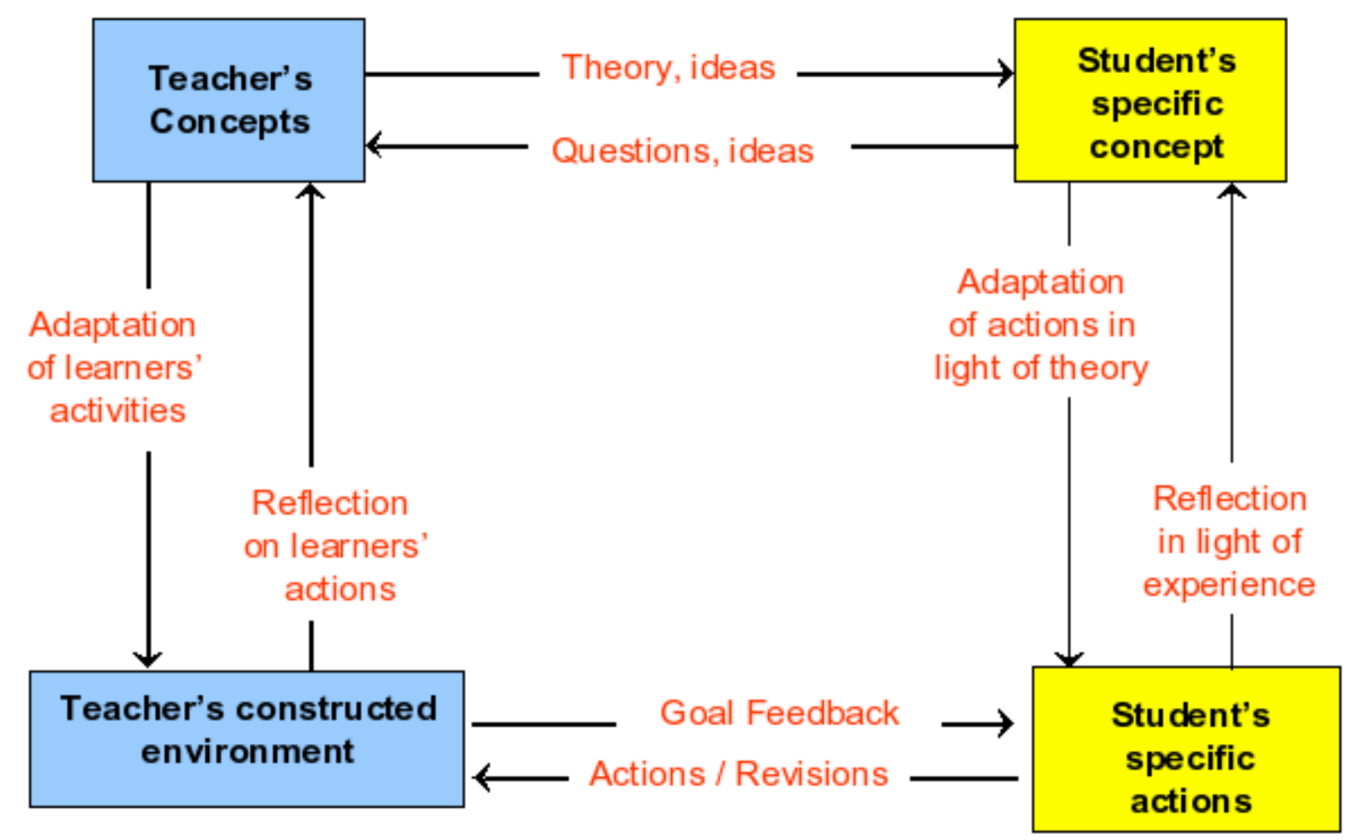

Figure 1: Laurilland's conversational educational media framework (Laurillard, 2013)

\subsubsection{Adaptation of the learning environment to suit all learners' actions}

The lecturer must teach the course content in a way that takes into consideration learners' existing knowledge conceptions and their knowledge as reflected from the results of the proficiency tests, and learners must incorporate the feedback and link it to their own knowledge conceptions. 


\subsubsection{Reflection on the learners' actions}

The lecturer should provide support to the learners in revising their knowledge conceptions and adapting learning to suit their learning needs. Learners should reflect on their learning process (initial concepts, tasks, objectives, feedback) and 'adapt' their learning actions, as a way of reconciling the theoretical knowledge and practical experiences. The model argues that interaction is at the centre of 'deep' learning (Laurillard, 2013) and that it helps learners to transform the knowledge gained by exploring beyond memorisation of the main concept being taught. Furthermore, 'deep' learning allows the learner to interact with the course material by creating relevant arguments and examples related to their daily lives.

Laurillard argues that only the use of "multimedia tutorial simulation" as an adaptive technology can meet this learning outcome (deep learning) (Clinch, 2005). Laurillard suggested five media forms that support various learning outcomes, which are: (a) narrative, (b) interactive, (c) communicative, (d) adaptive, and (e) productive. Narrative media show the learner virtual image or text, while interactive media such as search engines and randomised multiple-choice questions respond to the learners' actions. Communicative media such as email and discussion forum platforms facilitate communication exchanges. Adaptive media, such as simulation and virtual words, respond to the user action. The study on which this paper is based adopted an adaptive medium to grade web-based assignments and to provide immediate feedback to the learners. Furthermore, proficiency tests were used to monitor learners' learning activities.

\section{CONTEXT OF THE STUDY: USE OF WILEY PLUS ORION}

Physics 10th Edition by Cutnell and Johnson (1998), published by Wiley, is used as the prescribed textbook for the Physics course at the Cape Peninsula University of Technology. The online platform Wiley Plus has been used since 2014 in the Engineering Physics course at this university of technology (UoT) due to its ability to provide online assessment and timeous feedback to the learner. An addition to Wiley Plus is the ORION proficiency test, which helps the teacher to (a) gain insight into each individual student's level of proficiency and confidence level; (b) identify and tailor learning for individual students through adaptive practice; and (c) assist individual students during office hours on areas of improvement. The lecturer encouraged learners to use the "Read, Practice, Study" section on the online platform that contains an additional set of questions, videos of sample problems being solved, electronic text content, simulation activities and a solution manual for selected problem-set questions. Learners' activities on the Wiley Plus system's "Read, Practice, Study" section cannot be accessed by the lecturer to monitor their study habits. Hence, the introduction of ORION to the Wiley Plus package in 2014 (which allowed lecturers to access these sections) offered an efficient way of encouraging learners to study and provided learning support to the first-year Engineering Physics learners in the extended curriculum programme (ECP). The ECP programme has been designed to support learners who are enrolled in the Chemical Engineering programme with a 50 percent pass rate in high school (matric) Mathematics and Physical Sciences. To ensure that the ECP learners succeed in their studies, the learners take half a workload compared to mainstream learners 
(mainstream learners are those whose matric marks are above 50 percent and who take six subjects per semester) studying for the National Diploma in Chemical Engineering.

The lecturer responsible for teaching physics, with support from the Department and John Wiley \& Sons Publishers, piloted the Wiley PLUS ORION software to evaluate the effectiveness of the software for proficiency tests and provision of immediate feedback to learners, and hence enhancing learners' performance. Learners were encouraged to strive to get 50\% proficiency in each of the chapters assigned to them by the course instructor and at the end of the semester, the learners' scores were assessed and weighed 10\% of the semester mark. The effectiveness of the ORION proficiency test was evaluated through analysing feedback from three Wiley Plus ORION feedback reports, namely: 1) Metacognitive report, 2) Productivity report, and 3) The most challenging activity feedback report. Furthermore, the scores in the proficiency test were compared to the paper-based term test scores to see if the performance correlates.

\section{METHODOLOGY}

Both quantitative and qualitative approaches were used in this study. Quantitative approaches involve using scientific or mathematical data to understand a problem, while qualitative approaches entail non-measurable data such as focus group interviews.

\subsection{Context and participant}

The study was conducted at the Faculty of Engineering, Department of Chemical Engineering, at a South African university of technology. The participants of the study were 44 registered learners who were enrolled for the ECP Chemical Engineering Physics course in 2015 and who were studying towards a national diploma.

\subsection{Data collection}

The data was collected using WPO from John Wiley \& Sons Publishers. Three reports were drawn from the online system: 1) Metacognitive report, which compares learners' proficiency on WPO to learners' confidence level; 2) Productivity report, which compares time spent in WPO to learners' overall proficiency in WPO; and 3) The most challenging activity feedback report, which sorts objectives and chapters by proficiency, from lowest to highest.

Furthermore, learners' scores on the WPO proficiency test were compared to their scores on the paper-based midterm test to measure their performance on the WPO proficiency tests and its impact on the paper-based midterm test.

Three focus group interviews were conducted, one with each category of learners: high (with an average score of 68\%), middle (with an average score of 52\%) and lower performing (with an average score of $27 \%$ ) to gain insight into their experience with the WPO. 


\subsection{Data analysis}

Data derived from the WPO 1) Metacognitive report, 2) Productivity report, and 3) The most challenging activity feedback report was analysed using inferential statistics. The inferential statistics used was the Pearson product moment correlation coefficient (PPMC), which evaluates the strength of the correlation between WPO proficiency test scores compared to the paper-based midterm averaged test. Focus group interviews were analysed inductively to elicit learners' opinion on the use of the WPO proficiency tests.

\subsection{Ethical clearance}

The participants' consent to take part in the study was sought and the purpose of the study was explained to the learners. Interview transcripts and learner scripts were available for the learners to scrutinise. Anonymity and confidentiality were adhered to as promised to the learners. The Fundani Centre for Higher Education and Development ethics committee provided ethical clearance for the study.

\section{FINDINGS AND DISCUSSION}

\subsection{Analysis of learners' metacognitive report}

The metacognitive report compares learners' proficiency in WPO to their level of confidence on the subject matter. Metacognition is the "awareness and understanding of one's own thought process" (Heider, 2015). In Figure 2, the upper left quadrant on the graph indicates that $52 \%$ of learners had higher levels of proficiency in WPO, but lower confidence.

This result could partly be explained by learners' assertion during the focus group interviews that those who stayed outside campus with no access to a computer and the internet spend less time on the WPO proficiency tests. This may have resulted in them answering questions under pressure to meet the deadline and receiving help from their peers who reside on campus at the last minute and scoring high scores without awareness and understanding of their own thought process, hence leading to a lower confidence level, as indicated by both learner A and learner B:

Learner A: If like, when I was staying off campus and then I didn't have internet and stuff and so my friends, some of my friends stayed on campus and then they did, they spent a lot of time on Wiley Plus they did the questions and they, maybe it's going to be submitted later today and I haven't done all the work because I'm at home, I don't have internet access. I come on that day, I'm like okay, I'm going to do this and then they show me. I do it with them they say no, click this, click this, and click this. After less time, but I get good results. I've spent less time. I didn't even work out the problems. They just told me. [sic] 


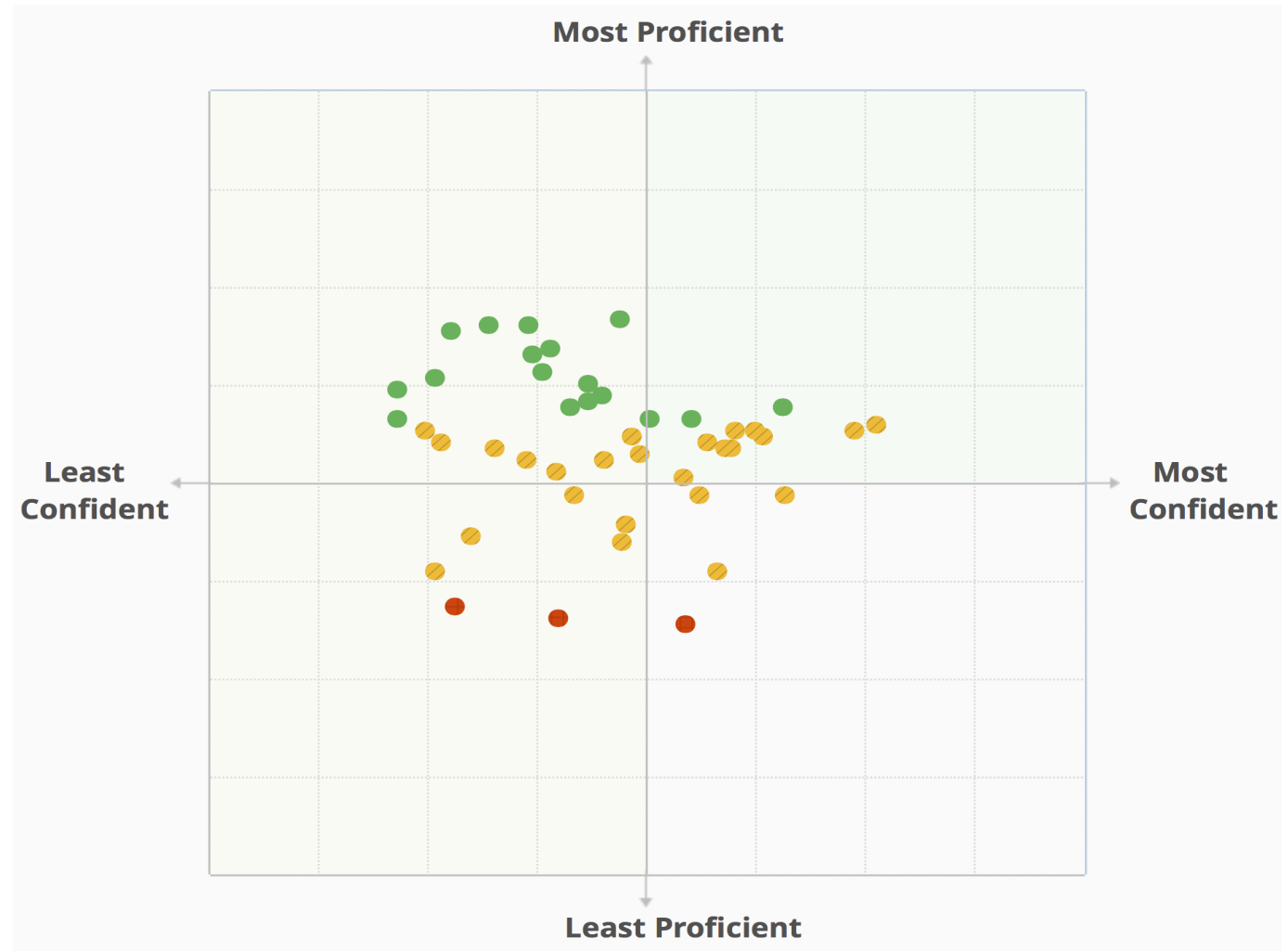

Figure 2: Learners' proficiency level compared to the level of confidence

Learner B: Because I was under pressure, it's going to close on me. I'm under pressure here, so just clicking, clicking what my friends are telling me and then I get good results[.] [sic]

In Figure 2, the lower left quadrant indicates that 14\% of the learners had low proficiency in WPO and low confidence (low-performing learners). These learners may have been underprepared and needed to spend more time working with the resources in WPO to boost their confidence and proficiency. A lack of access to computers/smart phones for learners residing off campus and unstable Wi-Fi for learners on campus may have contributed to learners' low scores and confidence level, as shown in the following quotes:

Learner C: Wiley Plus was fine but there are a little bit challenges for people who don't stay on campus. I suggest maybe Wiley Plus can be improved. You have some program, not online, because people who stay outside campus they struggle a lot because they don't have access to computer or smart phone.

Learner D: Because sometimes, Wi-Fi, it's boring. It will be offline the whole day so you 
won't study in Wiley Plus, you won't log in. I will say it should be an off-line system. Like you can download it then you work throughout without Internet.

The results also show that learners suggested that an offline WPO would be helpful for learners who reside off campus as they lack internet or data to access the online platform.

The upper right quadrant shows that $24 \%$ of learners had higher levels of proficiency and high confidence (high-performing learners). These learners did well in WPO. During the focus group interviews, some of the learners in this category of learners indicated that timeous feedback on how they responded to a question helped them in building confidence to carry on with other questions:

Learner E: It actually gives us confidence that you know, okay, I got this answer. It confirms and it actually gives me confident to carry on.

The lower right quadrant indicates that $10 \%$ of the learners had lower levels of proficiency in WPO, but higher levels of confidence (middle-performing learners). This group of learners may have needed to spend more time working with the resources in WPO in order to help them clarify some misconceptions.

\subsection{Analysis of learners' productivity reports}

The productivity report compares time spent in WPO by learners to their overall proficiency in WPO. Figure 3 shows that the average time spent by high-performing learners (that is, those with an average score of $68 \%$, shown in green dots) was 16 hours, while the middle-performing learners had an average score of $52 \%$ (shown in yellow) and spent 15 hours on the system. The low-performing learners had an average score of $27 \%$ and spent 10 hours on WPO.

From Figure 3 it is evident that learners who spent more time on WPO performed better compared to those who spent less time. Reasons for spending less time on WPO can be deduced from the following learners' quotes:

Learner F: Wiley Plus was fine but there are a little bit challenges for people who don't stay on campus. I suggest maybe Wiley Plus can be improved. You have some program, not online because people who stay outside campus they struggle a lot because they don't have access to computer or smart phone.

Learner G: Because sometimes, Wi-Fi, it's boring. It will be offline the whole day so you won't study in Wiley Plus, you won't log in[.]

A lack of computers/smart phones for learners staying off campus and unreliable Wi-Fi for learners residing on campus may have affected the time learners spent on Wiley Plus ORION. 


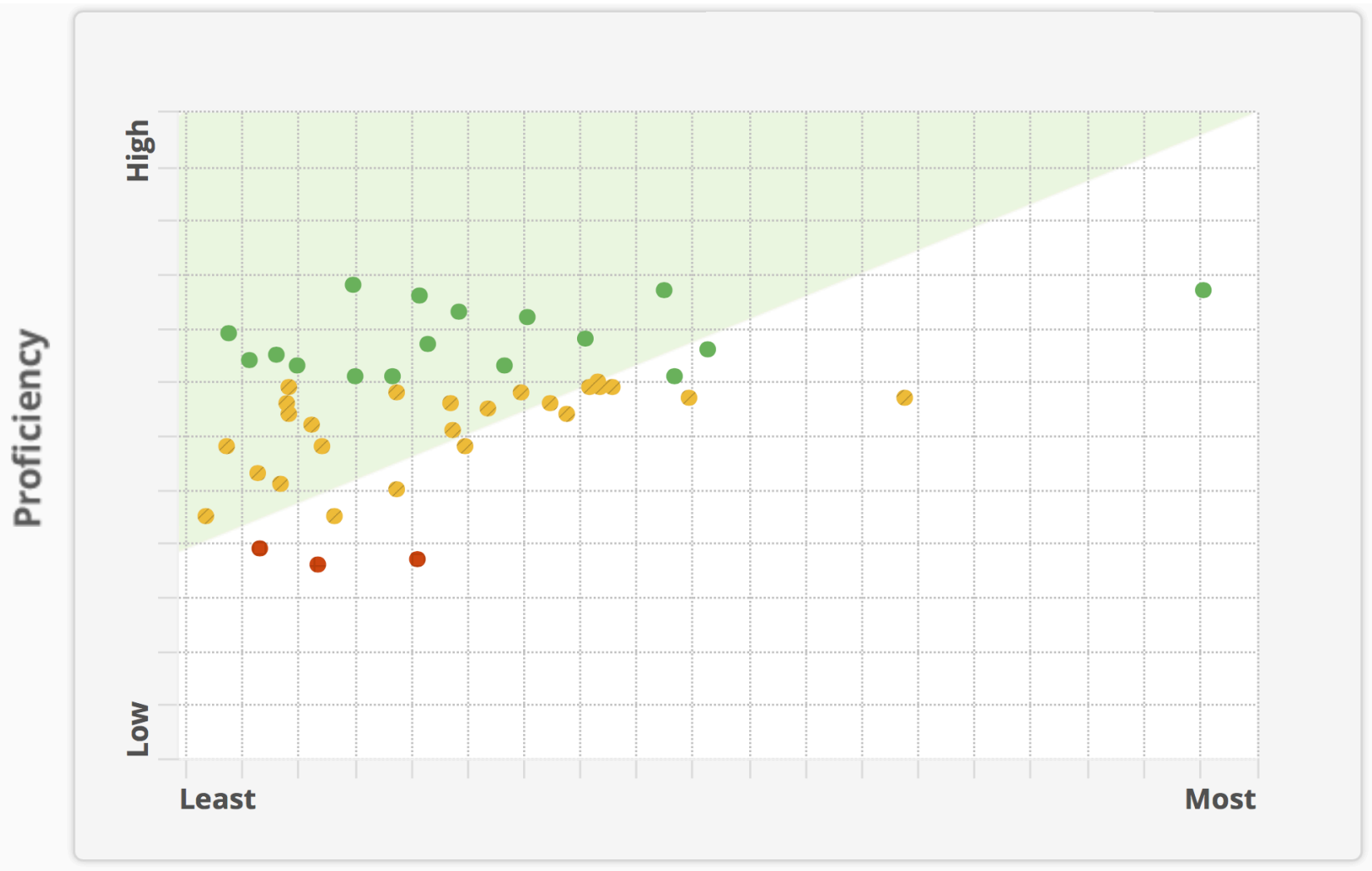

Time Spent

Figure 3: Learners' average proficiency score against time spent on Wiley Plus with ORION 


\subsection{Analysis of learners' most challenging activities feedback report}

The most challenging activities report in WPO sorts objectives and chapters by proficiency, from lowest to highest, as shown in Figure 4.

\begin{tabular}{|c|c|c|c|c|}
\hline View By Chapters & View By Objectives & & & \\
\hline \multicolumn{2}{|l|}{ E Chapters } & 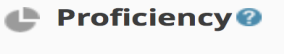 & $\underset{\text { Performance }}{\simeq}$ & Performance 8 \\
\hline \multicolumn{2}{|c|}{ Ch 12: Temperature and Heat } & 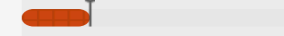 & $25 \%$ & $5 / 14$ \\
\hline \multicolumn{2}{|l|}{ Ch O: Math Skills } & $\infty i$ & $34 \%$ & $8 / 25$ \\
\hline \multicolumn{2}{|l|}{ Ch 11: Fluids } & is & $42 \%$ & $55 / 188$ \\
\hline \multicolumn{2}{|c|}{ Ch 13: The Transfer of Heat } & is & $47 \%$ & $16 / 32$ \\
\hline \multicolumn{2}{|c|}{ Ch 6: Work and Energy } & i & $47 \%$ & $66 / 252$ \\
\hline \multicolumn{2}{|c|}{ Ch 19: Electric Potential Energy and the... } & $i$ & $51 \%$ & $53 / 165$ \\
\hline \multicolumn{2}{|c|}{ Ch 7: Impulse and Momentum } & $i$ & $52 \%$ & $100 / 328$ \\
\hline \multicolumn{2}{|c|}{ Ch 5: Dynamics of Uniform Circular Mo... } & is & $54 \%$ & $73 / 228$ \\
\hline \multicolumn{2}{|c|}{ Ch 3: Kinematics in Two Dimensions } & is & $58 \%$ & $106 / 363$ \\
\hline \multicolumn{2}{|c|}{ Ch 20: Electric Circuits } & i & $60 \%$ & $145 / 238$ \\
\hline \multicolumn{2}{|c|}{ Ch 18: Electric Forces and Electric Fields } & i & $61 \%$ & $125 / 508$ \\
\hline \multicolumn{2}{|c|}{ Ch 1: Introduction and Mathematical C... } & i & $62 \%$ & $113 / 290$ \\
\hline \multicolumn{2}{|c|}{ Ch 2: Kinematics in One Dimension } & i & $62 \%$ & $103 / 369$ \\
\hline \multicolumn{2}{|c|}{ Ch 4: Forces and Newton's Laws of Mot... } & 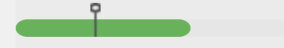 & $64 \%$ & $116 / 449$ \\
\hline \multicolumn{2}{|c|}{ Ch 8: Rotational Kinematics } & i & $65 \%$ & $22 / 54$ \\
\hline \multicolumn{2}{|c|}{ Ch 10: Simple Harmonic Motion and El... } & i & $66 \%$ & $10 / 48$ \\
\hline \multicolumn{2}{|c|}{ Ch 9: Rotational Dynamics } & i & $67 \%$ & $16 / 45$ \\
\hline
\end{tabular}

Figure 4: The most challenging activities report in Wiley Plus with ORION

It can be deduced from the figure that the chapters in which learners had attempted answering questions showed better learner improvement in scores compared to chapters where learners had not attempted questions.

\subsection{Learners' performance on Wiley Plus with ORION compared to performance on paper-based midterm averaged test}

The scatterplot shows that learners' average physics midterm test scores and the average WPO scores had a positive correlation (coefficient $=0.2098$ ). Using a k-means cluster analysis, the researchers identified three learner segments: high performing (20\% of learners), medium performing (35\% of the learners) and low performing (45\% of learners). Results showed that the high-performing 
learners had the highest averaged WPO scores of $52 \%$ and an average paper-based score of $66 \%$. The medium-performing learners had medium averaged WPO scores of $44 \%$ and averaged paper-based test scores of $45 \%$, while the low-performing learners had averaged WPO scores of $39 \%$ and averaged paper-based test scores of $29 \%$.

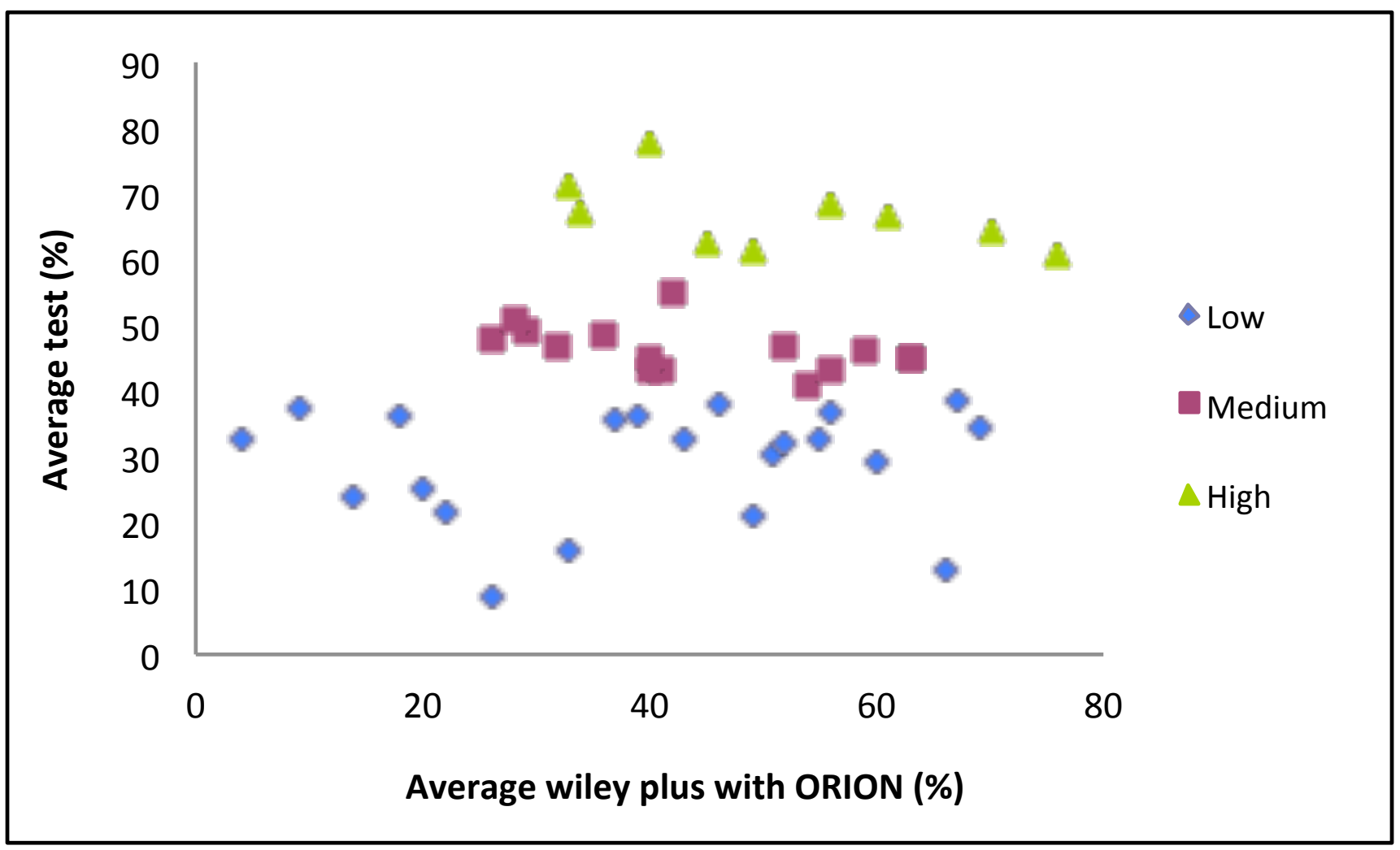

Figure 5: Average mid-test scores compared to Wiley Plus with ORION average proficiency test scores

\section{CONCLUSION}

The WPO was used in this study as an adaptive medium, supporting Laurilland's conversational framework for teaching and learning and her premise that learning is an active process including a cycle of "goal-action-feedback-reflection-adaptation-revision-feedback-reflection" (Laudrillard, n.d.). The findings showed that learners who spent more time on the WPO adaptive learning system performed better in their course than those who spent less time. For example, learners who spend more time on WPO attempting proficiency tests had a higher chance of performing much better on the paper-based midterm averaged test.

The metacognitive report showed that most of the learners who were most proficient showed least confidence in terms of their awareness and understanding of their own thought process. These 
learners were encouraged to spend more time on the WPO in order to maintain their proficiency and increase their confidence. The learners who showed a low level of proficiency and confidence were encouraged to spend more time working with WPO to help clarify some of the misconceptions they held. The most challenging activities report showed that in chapters where learners had attempted to answer more questions, there was a better improvement in learners' overall scores. The findings also showed that there was a positive correlation between the paper-based midterm averaged test and WPO proficiency test overall scores. Furthermore, WPO was shown to be an effective adaptive system as learners who spent more time on the system performed better in their course than those who used it for a shorter time. For example, learners who spend more time on WPO attempting proficiency tests had a higher chance of performing much better on the paper-based midterm averaged test.

\section{References}

Basitere, M. \& Ivala, E. N. (2015). The effects of Wiley PLUS web-based homework system on student performance in the Chemical Engineering Extended Curriculum Program introductory physics course. In International Conference on e-Learning (p. 31). Academic Conferences International Limited.

Basitere, M. \& Ivala, E. N. (2017). An evaluation of the effectiveness of the use of multimedia and Wiley PLUS web-based homework system in enhancing learning in the chemical engineering extended curriculum program physics course. Electronic Journal of e-Learning, 15(2), 156-173.

Beal, C. R., Qu, L., \& Lee, H. (2008). Mathematics motivation and achievement as predictors of high school students' guessing and help-seeking with instructional software. Journal of Computer Assisted Learning, 24(6), 507-514. https://doi.org/10.1111/j.1365-2729.2008.00288.x

Bonham, S. W., Deardorff, D. L., \& Beichner, R. J. (2003). Comparison of student performance using web and paper-based homework in college-level physics. Journal of Research in Science Teaching, 40(10), 1050-1071. https://doi.org/10.1002/tea.10120

Bonham, S., Beichner, R., \& Deardorff, D. (2001). Online homework: does it make a difference? The Physics Teacher, 39(5), 293-296. https://doi.org/10.1119/1.1375468

Clinch, P. (2005). Supporting law teaching: training and teaching. In UKCLE Seminar Teaching and learning for legal skills trainers. Last accessed: July 2005. Retrieved from http://www.ukcle.ac. uk/events/clinch.html

Corbett, A. T. \& Anderson, J. R. (1992). Student modeling and mastery learning in a computer-based programming tutor. In International conference on intelligent tutoring systems (pp. 413-420). Springer. https://doi.org/10.1007/3-540-55606-0_49

Cutnell, J. D. \& Johnson, K. W. (1998). Physics. Wiley.

Demirci, N. (2007). University students' perceptions of web-based vs. paper-based homework in a general physics course. Online Submission, 3(1), 29-34. https://doi.org/10.12973/ejmste/ 7537https://doi.org/10.12973/ejmste/753711

Eliot, C. \& Woolf, B. P. (1994). Reasoning about the user within a simulation-based real-time training system. In Fourth International Conference on User Modeling, Hyannis Cape Cod, Mass (pp. 1519). 
Gibbs, G. (2010). Using assessment to support student learning. Last accessed: 03 Oct 2017. Leeds Metropolitan Press. Retrieved from boo://goo.gl/1MsxU4

Hattie, J. \& Timperley, H. (2007). The power of feedback. Review of educational research, 77(1), 81-112. https://doi.org/10.3102/003465430298487

Hauk, S. \& Segalla, A. (2005). Student perceptions of the web-based homework program WeBWorK in moderate enrolment college algebra classes. The Journal of Computers in Mathematics and Science Teaching, 24(3), 229.

Heider, J. S. (2015). Using digital learning solutions to address higher education's greatest challenges. Publishing Research Quarterly, 31(3), 183-189. https://doi.org/10.3102/003465430298487

Jones, C. G. (2008). Student perceptions of the impact of web-based homework on course interaction and learning in introductory accounting. Issues in Information Systems, 9.

Koedinger, K. R., Anderson, J. R., Hadley, W. H., \& Mark, M. A. (1997). Intelligent tutoring goes to school in the big city. International Journal of Artificial Intelligence in Education, 30-43.

Laudrillard, D. (n.d.). Learning formal representations through multimedia. In F. Marton, D. Hounsell, \& N. Entwistle (Eds.), The experience of learning (pp. 172-183). Edinburgh: Scottish Academic Press.

Laurillard, D. (2013). Rethinking university teaching: a conversational framework for the effective use of learning technologies. Routledge.

McLaren, B. (2013). Adaptive online learning the present and future of education. Last checked: 03 Oct 2017. John Wiley \& Sons, Inc. Retrieved from http://www.wiley.com/college/sc/ oriondemo/pdf/Adaptive\%5FOnline\%5FLearning.pdf

Nicol, D. (2011). Good designs for written feedback to students. In M. Svinicki \& W. J. McKeachie (Eds.), Mckeachie's teaching tips: strategies, research and theory for college and university teachers (pp. 108-124).

Schreiber, S. J., Smith, K. J., \& Getz, W. M. (2014). Calculus for the life sciences. Wiley.

Shute, V. J. (2008). Focus on formative feedback. Review of educational research, 78(1), 153-189. https://doi.org/10.3102/0034654307313795

Vanlehn, K., Lynch, C., Schulze, K., Shapiro, J. A., Shelby, R., Taylor, L., ... Wintersgill, M. (2005). The Andes physics tutoring system: Lessons learned. International Journal of Artificial Intelligence in Education, 15(3), 147-204. 